\section{Public Health Genomics}

Public Health Genomics 2011;14:85-93

DOI: $\underline{10.1159 / 000294277}$
Received: July 7, 2009

Accepted after revision: January 6, 2010

Published online: March 18, 2010

\title{
Participation in Genetic Testing Research Varies by Social Group
}

\author{
Sharon Hensley Alford ${ }^{\mathrm{a}}$ Colleen M. McBride ${ }^{\mathrm{b}}$ Robert J. Reid ${ }^{\mathrm{d}}$ Eric B. Larson ${ }^{\mathrm{d}}$ \\ Andreas D. Baxevanis ${ }^{c}$ Lawrence C. Brody ${ }^{c}$ \\ ${ }^{a}$ Henry Ford Hospital, Detroit, Mich., ${ }^{b}$ Social and Behavioral Research Branch and ' Genome Technology Branch, \\ National Human Genome Research Institute, Bethesda, Md., and d Group Health Cooperative, Seattle, Wash., USA
}

\section{Key Words}

Multiplex genetic susceptibility test $\cdot$ Race $\cdot$ Gender $\cdot$

Education

\begin{abstract}
Background: Advances in technology have made individual access to personal genetic information foreseeable in the near future. Policy makers and the media forecast that the ready availability of personal genetic profiles would benefit both the individual and the health care system by improving outcomes and decreasing cost. However, there is a significant gap between having access to genetic data and either wanting or understanding the information it provides. $\mathbf{O b}$ jective: Our primary aim was to evaluate, using a population-based sample of healthy adults, whether gender, race and education status influences interest and participation in a multiplex genetic susceptibility test. Methods: Healthy, insured individuals, 25-40 years of age, were approached via a large, integrated health system in which primary and specialty care is available. Study participants were offered personalized genetic risk information on 8 common chronic health conditions. Social groups historically known not to participate in genetic research (men, African Americans and those from lower education neighborhoods) were oversampled. We describe the recruitment outcomes and testing de-
\end{abstract}

\section{KARGER}

Fax +4161306 1234

E-Mail karger@karger.ch

www.karger.com (c) 2010 S. Karger AG, Basel

Accessible online at: www.karger.com/phg cisions of these social groups. Results: We found that even among those with access to health care, African Americans were less likely to participate in the multiplex genetic susceptibility test, while those from higher education neighborhoods were more likely to participate. Conclusions: Our results suggest that large social groups will likely be underrepresented in research in personalized genomics even when robust population-based recruitment strategies are employed.

Copyright $\odot 2010$ S. Karger AG, Basel

\section{Introduction}

Discoveries stemming from the completion of the sequencing of the human genome, results of the HapMap project [1] and the burgeoning growth of genome-wide association studies [2] have fueled the new field of personalized genomics. The future for the field is proffered as one in which individuals and health care providers might use genomic risk information to facilitate decisionmaking, personalize treatment approaches and motivate lifestyle improvements and adherence to screening recommendations $[3,4]$.

Multilocus genetic testing is a tool now commonly suggested as a means to actualize the vision of personal- 
ized genomics [5, 6]. This tool and others (e.g. whole genome sequencing) enable individual genetic risk profiles to be generated which, in the future, could be used to tailor specific prevention recommendations [7].

In the USA and other developed countries, several commercial entities are marketing genome-wide singlenucleotide polymorphism-based tests directly to the public $[8,9]$. After purchasing a test, the consumer is provided access to highly interactive Web sites that present specific information about their genome, including ancestry, personal characteristics and genotype information. Although not directly sold as a medical test, these products also provide estimates of disease risk. Rapid advancements in technology suggest that the price of such testing will become increasingly affordable (and therefore attainable).

As with other advanced technologies, concerns have been raised that all segments of the population may not have equal access to personalized genomics. In particular, several studies have suggested that minority groups, such as African Americans and those with low levels of education, may not be 'reached' by these technologies [10-13]. This may be due to the fact that these individuals will not be approached to consider these services or that some groups may be less accepting of genomic products due to concerns about privacy or distrust in the medical system. Genetic research in the USA to date (largely in the area of inherited cancers) has shown that minority groups and the less educated are underrepresented even when extra efforts have been made to facilitate broadbased recruitment $[14,15]$. Moreover, men are frequently underrepresented in genetics research. This has been observed even in studies such as those for familial colorectal cancer, where risk is not influenced by gender [16].

An added factor that may contribute to differential access to new technologies is the increasing trend to distribute health information via the internet. Internet access to health information can arguably empower the public as health consumers since it enables user-controlled interactions and is available virtually free of charge [17]. There is still concern, however, that information providers may widen the 'digital divide' if they rely solely on the internet for information distribution. Certain segments of the population still do not have convenient access to the internet and/or may distrust the security of information shared online [18]. Studies to date have shown that women, whites and the highly educated are more likely to use the internet to obtain health information [19].

It is difficult to predict how the US public will respond to offers of genetic testing given that the majority of ge- netic testing research has been carried out in the setting of familial disease. Recruitment into inherited cancer protocols has been based primarily in tertiary care centers, such as cancer centers at academic medical centers, and has either relied on volunteers or snowball sampling techniques [20]. To our knowledge, to date, there has been no population-based sampling strategy used to rigorously evaluate observed demographic differences in participation in multiplex genetic research and tests. This becomes particularly important as we enter the era of personalized genomics because it can be applied to common chronic disease risk, which has relevance to the entire population.

To better understand how genetic testing will be perceived by the US public, we launched the Multiplex Initiative, a preclinical, prospective, observational study in which study participants would be provided with information about genetic testing for common health conditions and be given the opportunity to receive a genetic test and personalized risk report. Our primary aim (and the focus of this report) was to evaluate, using a population-based sample of healthy adults (i.e. a sampling frame with a known denominator), whether gender, race and education status influence interest and participation in a multiplex genetic susceptibility test. To answer this question, we surveyed a representative sample of healthy adults and offered them educational materials about genetic susceptibility testing and an opportunity to undergo a free genetic test. The study was designed to minimize known barriers to participation and purposely oversampled groups historically underrepresented in genetic research. Our approach is unique compared to prior genetic testing studies in that we explore the participation of African Americans in genetic testing for low-penetrance, high-prevalence genes in a sample with a known denominator.

\section{Methods}

We designed a population-based, prospective, observational study and recruited participants who were enrolled with the Henry Ford Health System (HFHS) in Detroit, Mich., USA. The HFHS comprises the Henry Ford Medical Group (HFMG), which is a large medical group practice, as well as the Health Alliance Plan (HAP), which is a health maintenance organization. Our study population was drawn from a pool of approximately 350,000 patients at the intersection of HFMG-HAP. When patients first visit the health system, they are provided a copy of the systems 'Notice of Privacy Practices'. The notice includes explanations of the use of personal information for approved research. A master patient index is maintained with enrollment and basic demographic in- 
Table 1. Sampling distribution by gender, race and education neighborhood level

\begin{tabular}{lllllll}
\hline & \multicolumn{2}{l}{ African American } & & \multicolumn{2}{l}{ White } & \\
\cline { 2 - 3 } \cline { 5 - 6 } & Men & Women & & Men & Women \\
\hline High education neighborhood & $910(14)$ & $762(12)$ & & $645(10)$ & $568(9)$ \\
Low education neighborhood & $1,150(18)$ & $918(14)$ & & $763(12)$ & $632(10)$ \\
\hline
\end{tabular}

The total number in the sample was 6,348. Values represent numbers of people (percentages of the total sample in parentheses).

formation. From this list we were able to identify study subjects who had been enrolled for at least 1 year in HAP, were aligned with a HFMG primary care provider and were between $25-40$ years of age. Individuals whose electronic medical record included an ICD-9-coded clinical appointment for diabetes mellitus (type 1 or 2), atherosclerotic cardiovascular disease or osteoporosis were excluded. HFHS is part of the Detroit Surveillance, Epidemiology and End Results (SEER) tumor registry and has been participating since the registry's inception in 1973 . This registry was matched to the pool of potential participants to exclude individuals with a prior history of cancer (except non-melanoma skin cancer).

As men and African Americans have been underrepresented in prior genetic research studies, we oversampled individuals from these groups. We used the master patient index data to identify patients' self-reported gender and race. The initial sample was $59 \%$ African American versus $41 \%$ white. We also oversampled those predicted to have less education. To approximate education status, we mapped the patient's address to information from the 2000 US Census. Individuals from census block groups where $10 \%$ or more of the residents whose educational level was high school or below were considered to be from 'low education neighborhoods' and likely to be of a lower education status themselves. By this method, $55 \%$ of people in our sample were from low education neighborhoods.

Using this oversampling scheme, we created a participant file containing 6,600 potential participants. We accepted only 1 individual from each household. In a staggered fashion, we mailed advance letters advising the potential participants that they would be asked to participate in a survey. A USD 2 bill was included with this mailing and a toll-free number to call if the potential participant did not want to participate. Approximately 2 weeks after the advance letter was mailed and the participant had not called to decline participation, the Center for Survey Research at Group Health Cooperative (Seattle, Wash., USA) telephoned each participant to conduct a 35-min baseline interview. The survey included confirmation of the participant's medical history as well as psychological and behavioral assessments. Those who self-reported a personal medical history of conditions that excluded them from the study (e.g. diabetes, heart disease and osteoporosis) were given an abbreviated survey and excluded from further participation in the study. At the conclusion of the survey, eligible interviewees were sent a brochure describing the Multiplex study.

After observing initial participation rates for 6 weeks, we increased study incentives to achieve 2 objectives, namely to increase overall recruitment and to encourage those who were not interested in testing to log on to review and evaluate the information provided about the Multiplex test. We added text to the survey's exit script specifically mentioning that we would be mailing a brochure about the next phase of the study with a USD 20 bill. In addition, we added exit script text that also mentioned the incentives available through the study's Web site. The cover letter text also mentioned the incentives available for completing the Web-based assessments. Finally, we asked for participants' e-mail addresses and sent them e-mail reminders that included a link to the study Web site. The brochure mailing included the study identifier and directions for logging into the Multiplex Web site. In the brochure, participants were offered a paper copy of the Web site, if they so desired. The Web site included information modules on genetic testing, specifics about the genes being tested and 4 questionnaires. Incentives (in the form of gift certificates to a major retailer) were offered for completing each assessment on the Web site, up to a total of USD 50 for completing all Web assessments. The focus of the first 3 assessments was to assess understanding of the information presented. The fourth assessment was simply one question, i.e. 'Are you interested in genetic testing?' Three responses were offered: 'Yes', 'No' and 'Maybe'. Participants who answered 'Yes' or 'Maybe' were contacted by a research educator (RE) to answer any additional questions and/or to schedule a clinic visit for blood collection.

To maximize participant convenience, multiple HFHS clinics throughout metropolitan Detroit served as sites for participants to provide written consent and blood samples. During the clinic visit, the RE used a PowerPoint presentation to explain the pros and cons of testing prior to obtaining the participant's written consent. After the presentation, the participant completed a questionnaire to evaluate their understanding of the content presented. The participant then signed the consent and provided a blood sample for the genetic test.

Both the National Institutes of Health and HFHS Institutional Review Boards approved all aspects of this study.

Comparisons were made between participation in each of the 3 major research contacts (i.e. completing the baseline survey, visiting the study Web site and having the genetic test performed) by each of our oversampled groups (race, gender, and education neighborhood). Comparisons were made using $\chi^{2}$ tests and quantified with logistic regression modeling. All $\mathrm{p}$ values were assigned using two-tailed tests. Logistic regression models were used for multivariate analyses. 
Fig. 1. Participant flow diagram. Asmt $=$

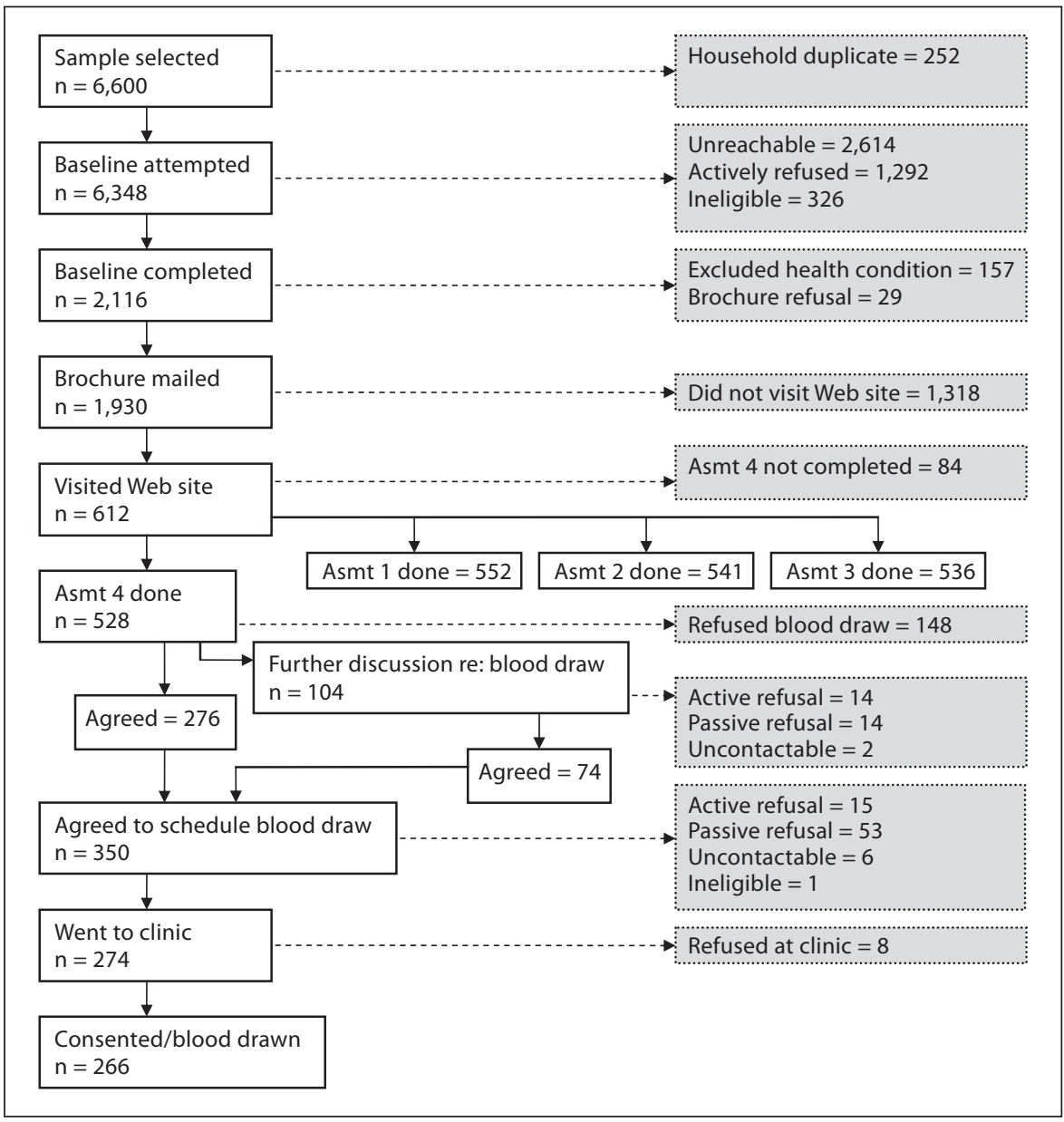
Assessment.

\section{Results}

\section{Baseline Survey Completion}

Contact information was identified for 6,600 participants potentially eligible for the study. The sampling distribution is described in table 1 . A total of 252 individuals were removed from the sampling frame because they lived in the same household as another individual selected for the study.

Figure 1 provides a diagram of the flow of participants through the study. Baseline surveys were attempted for 6,348 individuals, of whom 2,614 (42\%) were unreachable by phone, 1,307 (21\%) declined to participate and $326(8 \%)$ were determined to be ineligible for reasons such as age, language barrier or living out of the area. Another 157 individuals were determined to be ineligible during the survey due to self-reported history of an excluded health condition. Baseline surveys were completed with 2,116 (59\%) of the 3,577 surveyed individuals who were eligible.
Analysis by social group showed that men were significantly less likely to complete the baseline survey than women ( 29 vs. $39 \%$; p $<0.0001$; table 2), while African Americans were slightly but significantly less likely to complete the baseline survey than whites (33 vs. $36 \%$; $p=$ $0.03)$. Similarly, those living in a lower education neighborhood were also slightly but significantly less likely to complete the survey than those living in a higher education neighborhood (32 vs. 35\%; $\mathrm{p}=0.01$ ). Despite the lower rates of participation by these demographic groups, $57 \%$ of those who completed the baseline survey were African American and 52\% were from low education neighborhoods.

\section{Visiting the Web Site to Consider Testing}

Only 29 (1\%) of the individuals who completed the baseline survey declined the recruitment brochure mailing; therefore, brochures were mailed to 1,930 individuals. A third of those who received the brochure $(n=612)$ 
Table 2. Participation in the baseline survey, viewing of the study Web site and test participation by gender, race and education neighborhood

\begin{tabular}{|c|c|c|c|c|c|c|c|c|c|}
\hline & \multicolumn{3}{|l|}{ Gender } & \multicolumn{3}{|l|}{ Race } & \multicolumn{3}{|c|}{ Neighborhood education } \\
\hline \multicolumn{10}{|l|}{ Baseline survey } \\
\hline Invited & 3,468 & 2,880 & & 2,641 & 3,717 & & 3,463 & 2,885 & \\
\hline Completed & $1,003(29)$ & 1,113 (39) & 0.0001 & $949(36)$ & $1,237(33)$ & 0.03 & $1,104(32)$ & $1,012(35)$ & 0.01 \\
\hline Completed & 267 (29) & 345 (34) & 0.05 & $327(40)$ & $285(26)$ & $<0.0001$ & $325(32)$ & $287(31)$ & 0.60 \\
\hline \multicolumn{10}{|l|}{ Testing } \\
\hline Invited & 267 & 345 & & 327 & 285 & & 325 & 287 & \\
\hline Completed & $117(44)$ & $149(43)$ & 0.88 & $180(55)$ & $86(30)$ & $<0.0001$ & $132(41)$ & $134(47)$ & 0.13 \\
\hline
\end{tabular}

Values represent numbers of participants (percentages in parentheses). p values generated by $\chi^{2}$ comparisons of participation.

visited the Web site at least once. Of those who participated in the baseline survey that did not mention the Web assessment incentives or include the USD 20 bill with the brochure, $23 \%$ visited the Web site; this rate was significantly lower ( $p$ value $<0.0001$ ) than the $36 \%$ who were told about the incentives and were sent a USD 20 bill with their brochure. An additional automated e-mail reminder did not increase Web site visits, as 35\% who received the e-mail visited the Web site.

Among the 612 who visited the Web site at least once, the majority completed 1 or more of the $4 \mathrm{Web}$ site assessments $(90,88,88$ and $86 \%$ completed assessments $1-4$, respectively). Virtually everyone who completed assessment 4 (527/528) completed all 4 assessments. Fifty-eight individuals who visited the Web site did not complete any of the assessments. A single participant requested a paper copy of the Web site information and completed the online assessments by telephone.

Comparison of the likelihood of visiting the Web site at least once showed that social group, gender and race were significant predictors (table 2). Men were less likely to visit the Web site than women ( 29 vs. $34 \%$; $p=0.05$ ), and African Americans were less likely to visit the Web site than whites ( 26 vs. $40 \%$; $<<0.0001$ ). However, there was no difference according to education neighborhood status.

\section{Testing Decision}

Of the 528 individuals who completed assessment 4, i.e. the question regarding interest in testing, 276 responded 'Yes' that they wanted to be tested, 148 responded 'No' to decline testing and 104 responded 'Maybe'. We
Table 3. Comparison of decisive versus indecisive responses to the question regarding interest in genetic testing

\begin{tabular}{llll}
\hline & Decided & Undecided & p value \\
\hline $\begin{array}{llll}\text { Gender } \\
\quad \text { Men }\end{array}$ & $182(43)$ & $48(46)$ & \\
$\quad$ Women & $242(57)$ & $56(54)$ & 0.55 \\
$\begin{array}{l}\text { Race } \\
\quad \text { White }\end{array}$ & $256(60)$ & $40(38)$ & \\
$\quad$ African American & $168(40)$ & $64(62)$ & $<0.0001$ \\
$\begin{array}{l}\text { Education neighborhood } \\
\quad \text { Low }\end{array}$ & $221(52)$ & $59(57)$ & \\
$\quad$ High & $203(48)$ & $45(43)$ & 0.40 \\
\hline
\end{tabular}

Values represent numbers of participants (percentages in parentheses). $\mathrm{p}$ values generated by $\chi^{2}$ tests.

compared those who 'decided' outright about testing ('Yes' or 'No') to the 'undecided' ('Maybe') according to gender, race and education neighborhood (table 3). Decision status was significantly associated only with race. Whites were 2.5 times more likely than African Americans [odds ratio (OR) 2.44, 95\% confidence interval (CI) 1.57-3.79; table 3] to respond definitively 'Yes' or 'No' to the offer of genetic testing based on the information presented on the Web site.

A RE attempted to contact all 380 individuals who responded either 'Yes' (to schedule a clinic visit) or 'Maybe' (to provide additional information as needed). Of the 104 who responded 'Maybe', the majority (71\%) decided to be 
Table 4. Odds ratios [unadjusted (OR) and adjusted (aOR)] with 95\% CIs for participation in the baseline survey, visiting the study Web site and test participation

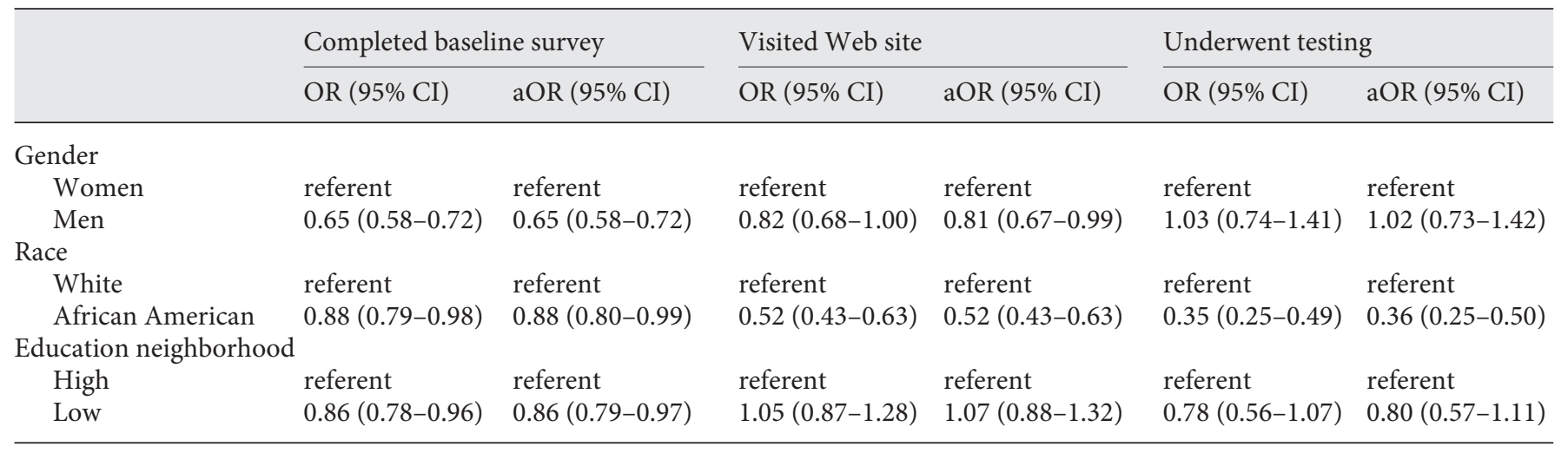

tested after speaking with a RE. However, African Americans remained significantly less likely than whites to seek testing even after talking with the RE ( 32 vs. 61\%; $\mathrm{p}=0.02)$.

Of the 350 who agreed to schedule a clinic visit for provision of consent and blood collection, 274 (77\%) actually came to the clinic. Eight of these individuals declined to be tested after meeting with the RE and viewing the presentation of the pros and cons of testing. Of the 30 individuals who were interested but did not keep their appointments, 14 refused when contacted for rescheduling, an additional 14 had a working phone number but either put off or did not answer our calls to schedule a clinic visit (i.e. passive refusal), and we were unable to find a current working number for 2 individuals.

Comparisons by social group of those who did and did not request testing showed only race as a significant predictor. African Americans were significantly less likely to be tested than whites ( 30 vs. $55 \%$; $p<0.0001$; table 2 ).

\section{Multivariate Comparisons for Completing the}

Baseline Survey, Visiting the Study Web Site and Making a Test Decision

We tested for pairwise interactions between gender, race and education neighborhood for each of the outcomes. None of the interactions were significant at the $\mathrm{p}<0.20$ level used to judge a significant interaction. Using logistic regression, we modeled the unadjusted and adjusted OR (OR and aOR, respectively) with 95\% CIs for each of the outcomes with gender, race and education neighborhood.

ORs were calculated for each of the 3 outcomes measured (table 4). There was virtually no difference between the univariate and multivariate analyses of each outcome. In multivariate analyses, men remained significantly less likely to complete the baseline survey than women (aOR $0.65,95 \%$ CI 0.58-0.72). Compared to a high education neighborhood, low education neighborhood status was associated with a $15 \%$ decrease in baseline survey participation (aOR 0.86, 95\% CI 0.79-0.97). African Americans were also significantly less likely to participate in the baseline survey (aOR 0.88, 95\% CI 0.79-0.99).

Men and African Americans were significantly less likely to visit the Web site (aOR $0.81,95 \%$ CI $0.67-0.99$, and aOR $0.52,95 \%$ CI $0.43-0.63$, respectively). There was no association between education neighborhood level and either visiting the Web site or getting tested. Race was the only factor significantly associated with participation in genetic testing. African Americans were significantly less likely to agree to testing than whites (aOR 0.35, 95\% CI 0.25-0.49).

\section{Discussion}

Our results show that men and African Americans, i.e. those typically underrepresented in health research, were also less likely to participate in personalized genetics research. As with any population-based approach, a sizable proportion of the individuals covered by the sampling frame were not reached despite earnest effort. While these results are consistent with prior research conducted within the context of familial cancers and clinical genetic testing in high-risk individuals [14, 15], our population-based approach with a known denominator lends considerable confidence to these findings. 
We were able to invite at least 3,000 or more members from these hard-to-reach groups. It is also noteworthy that lower participation in the research and testing among these social groups occurred in a context where several known barriers to participation were eliminated. Individuals could review information about testing online at their convenience, the test was free of charge and the target group had health insurance that provided access to preventative health care. Accordingly, even free genetic testing offered to individuals with health insurance will likely not be equally appealing to some groups of the US population.

It is possible that the observed differences across social groups with regard to test participation were influenced by our use of the internet as a decision aid. Those with lower education have been consistently shown to be less likely to have access to the internet [20]. Moreover, African Americans who visited the Web site were more likely than whites to respond 'Maybe' when queried about their interest in the free testing, suggesting that the absence of human interaction may have diminished willingness to commit to testing online. These individuals who responded 'Maybe' were later able to talk one-on-one with a RE. However, despite this personal contact to address questions and concerns, undecided white participants continued to be more likely to be tested than undecided African Americans. A recent review of participation in genetic testing among African Americans [21] suggests that African Americans may have less trust in researchers and health care organizations with respect to how genetic information might be used. To gain insight into this, we compared African Americans and whites on their responses to a number of questions about their relationship with their HFHS health care provider. We found a statistically significant difference in only one area; African Americans were less likely than whites to report that their doctor had spent enough time with them $(p=0.02$; Appendix). Thus, it does not appear that African Americans had any resounding distrust in the representatives of the health care system. However, their trust may not extend to health information conveyed through the internet or to researchers, for which we have no information.

We found that men were significantly less likely than women to visit the Web site and consider testing. Studies carried out on familial cancers have reported similar responses. Some research suggests that women are the family historians and purveyors of family history information and the gatekeepers for genetic testing [16]. However, consideration of the characteristics of the men in our study showed there was no difference in marital status between men who did and did not visit the Web site (51 vs. $55 \%$, respectively; $\mathrm{p}=0.77$ ).

The results reported here should be considered with the following caveats. Our measure of education neighborhood was a gross measure that likely represents considerable lumping together of educational groups. It is, however, very interesting to note that education neighborhood did not predict whether an individual would visit the Web site (32\% of those residing in high education areas viewed the Web-based material vs. $31 \%$ of those in low education neighborhoods; $\mathrm{p}=0.60$ ). This suggests, perhaps, that internet access and use was not a significant barrier to participation in the study. There also was not a significant difference in education neighborhood among those who participated in testing (41\% with high education vs. $47 \%$ low education; $\mathrm{p}=0.13$ ).

The baseline survey, in order to be as general as possible, did not include measures of receptivity to genetic testing that might have enabled us to delve deeper into the observed differences in participation according to social group. Unfortunately, we could not take advantage of preexisting information channels between potential study subjects and their health care providers. To maintain confidentiality, we purposely did not contact the participant's primary care provider, and no record of genetic testing was maintained at HFHS. This precluded the participation of primary care providers as agents or partners in the study. Toward the end of our study, the Genetic Information Nondiscrimination Act was signed into law [22]. This Act provides protection against health insurance discrimination based on gene status. This new law may allow future studies of genetic testing to be carried out in the context of routine health care.

The present study's reliance on the internet as a decision aid may have affected participation in a few different ways. Indeed, our previous report [23] showed that those who were savvy internet users were most likely to log on to consider testing. Moreover, some demographic groups such as those with low education may be especially unlikely to have access to or feel comfortable using the internet. Our findings also suggest that African Americans may have been reticent to make a decision based on the internet information alone. Additionally, offering the test with less demand for personal information disclosure prior to testing and/or offering a mail-in buccal/spit sample instead of a clinic visit for blood draw likely would have changed the makeup of our participants.

Despite these limitations, our population-based recruitment outcomes support the need to consider the likelihood that large social groups will be underrepresented 
in research relating to applications and translation of personalized genomics. Indeed, future research designs will likely need to include novel recruitment and oversampling approaches to arrive at externally valid results. Recent recruitment experiences of the Cancer Genetics Network [17] have identified several categories of promising minority recruitment strategies, such as identifying and recruiting at-risk families (e.g. those with strong family histories of health conditions), use of culturally tailored recruitment materials, aligning goals of research teams with priorities of community partners, involving members of the community in the research team and employing flexible strategies that address the needs of the community or individual participants. These methods could be evaluated alone or in combination in comparative effectiveness trials to identify optimal combinations of strategies that maximize the representation of diverse demographic groups in genetic testing research.

\section{Acknowledgements}

This work was supported by the Intramural Research Program of the National Human Genome Research Institute. However, the proposed research was made possible by collaboration with the Cancer Research Network funded by the National Cancer Institute (U19 CA 079689). Additional resources were provided by the Group Health Research Institute and Henry Ford Hospital. We are indebted to G. Gibney, D. Kanney, M. Fredricksen, D. Leja and C. Wade at the National Human Genome Research Institute; $H$. Kromei, E. Hasiec, N. Maddy, K. Wells and K. Husar at the HFHS, and C. Weise and R. Pardee at the Group Health Research Institute for their assistance and dedication to these studies. Genotyping services were provided by the Center for Inherited Disease Research. The Center for Inherited Disease Research is fully funded through a federal contract from the National Institutes of Health to The Johns Hopkins University (HHSN268200782096C). Our thanks also go to the study participants, who were all members of the HFHS.

\section{Appendix}

Health care service variables for those African American participants who did and did not visit the study Web site

\begin{tabular}{llll}
\hline & Visited & Did not visit \\
Web site & Web site & value \\
\hline Have regular personal doctor $^{1}$ & $0.78 \pm 0.42$ & $0.79 \pm 0.41$ & 0.77 \\
Often see personal doctor $^{2}$ & $2.18 \pm 0.94$ & $2.08 \pm 0.88$ & 0.14 \\
Personal doctor listens to you $^{3}$ & $5.45 \pm 1.04$ & $5.40 \pm 1.01$ & 0.54 \\
Clear instructions from doctor $^{3}$ & $5.50 \pm 1.04$ & $5.44 \pm 1.07$ & 0.51 \\
Doctor knows your medical history $^{3}$ & $5.28 \pm 1.05$ & $5.23 \pm 1.17$ & 0.59 \\
Doctor spends enough time with you $^{3}$ & $5.29 \pm 1.11$ & $5.05 \pm 1.34$ & 0.02 \\
Feel you can talk to doctor about anything $^{3}$ & $4.88 \pm 1.61$ & $4.73 \pm 1.63$ & 0.28 \\
Doctor gives you help that is needed $^{3}$ & $1.86 \pm 1.18$ & $1.88 \pm 1.18$ & 0.87 \\
Religion impacts health decisions $^{3}$ & $3.30 \pm 1.71$ & $3.16 \pm 1.63$ & 0.25 \\
\hline
\end{tabular}

Results are shown as means and standard deviations.

${ }^{1}$ Mean of responses 1 (yes) and 0 (no). ${ }^{2}$ Mean of responses 1 (once), 2 (twice) and 3 ( 3 or more times) for the last 12 months. ${ }^{3}$ Mean of responses on a Likert scale from 1 (never) to 6 (always).

\section{References}

1 The International HapMap Consortium: A second generation human haplotype map of over 3.1 million SNPs. Nature 2007;449:851861.

2 Guessous I, Gwinn M, Khoury MJ: Genomewide association studies in pharmacogenomics: untapped potential for translation. Genome Med 2009;1:46.
Khoury MJ, Feero WG, Reyes M, Cirtrin T, Freedman A, Leonard D, Burke W, Coates R, Croyle R, Edwards K, Kardia S, McBride C, Manolio T, Randhawa G, Rasooly R, St. Pierre J, Terry S; GAPP Net Planning Group: The Genomic Applications in Practice and Prevention Network. Genet Med 2009;11: 488-494.
4 US Department of Health and Human Services: Personalized Health Care: Pioneers, Partnerships, Progress. Washington, US Department of Health and Human Services, 2008. http://www.hhs.gov/myhealthcare/ news/phc_2008_report.pdf.

5 Katsanis SH, Javitt G, Hudson K: Public health. A case study of personalized medicine. Science 2008;320:53-54. 
6 Feero WG: The genome gets personal - almost. JAMA 2008;299:1351-1352.

$\checkmark 7$ Pharoah PD, Antoniou AC, Easton DF, Ponder BA: Polygenes, risk prediction, and targeted prevention of breast cancer. N Engl J Med 2008;358:2796-2803.

-8 Gollust SE, Hull SC, Wilfond BS: Limitations of direct-to-consumer advertising for clinical genetic testing. JAMA 2008;288:17621767.

9 Gurwitz D, Bregman-Eschet Y: Personal genomics services: whose genomes. Eur J Hum Genet 2009;17:883-889.

-10 Armstrong K, Micco E, Carney A, Stopfer J, Putt M: Racial differences in the use of BRCA1/2 testing among women with a family history of breast or ovarian cancer. JAMA 2005;293:1729-1736.

11 Lorence D, Park H: Group disparities and health information: a study of online access for the underserved. Health Informatics J 2008; 14:29-38.

12 Lorence DP, Park H, Fox S: Racial disparities in health information access: resilience of the digital divide. J Med Syst 2006;30:241249.
13 Moorman PG, Skinner CS, Evans JP, Newman B, Sorenson JR, Calingaert B, Susswein L, Crankshaw TS, Hoyo C, Schildkraut JM: Racial differences in enrolment in a cancer genetics registry. Cancer Epidemiol Biomarkers Prev 2004;13:1349-1354.

14 Ford BM: Factors associated with enrollment in cancer genetics research. Cancer Epidemiol Biomarkers Prev 2006;15:1355-1359.

15 Wonderling D, Hopwood P, Cull A, Douglas F, Watson M, Burn J, McPherson K: A descriptive study of UK cancer genetics services: an emerging clinical response to the new genetics. Br J Cancer 2001;85:166-170.

16 Koehly LM, Peterson SK, Watts BG, Kempf KK, Vernon SW, Gritz ER: A social network analysis of communication about hereditary nonpolyposis colorectal cancer genetic testing and family functioning. Cancer Epidemiol Biomarkers Prev 2003;12:304-313.

17 James RD, Yu JH, Henrikson NB, Bowen DJ, Fullerton SM; Health Disparities Working Group: Strategies and stakeholders: minority recruitment in cancer genetics research. Community Genet 2008;11:241-249.

18 Bernhardt JM, Lariscy RA, Parrott RL, Silk KJ, Felter EM: Perceived barriers to Internetbased health communication on human genetics. J Health Commun 2002;7:325-340.
19 Bernhardt JM, McClain J, Parrott RL: Online health communication about human genetics: perceptions and preferences of Internet users. Cyberpsychol Behavior 2004;7:728733.

20 Atkinson NL, Saperstein SL, Pleis J: Using the internet for health-related activities: findings from a national probability sample. J Med Internet Res 2009;11:e4.

21 Sterling R, Henderson GE, Corbie-Smith G: Public willingness to participate in and public opinions about genetic variation research: a review of the literature. Am J Public Health 2006;96:1971-1978.

22 Hudson KL, Holohan MK, Collins FS: Keeping pace with the times - the Genetic Information Nondiscrimination Act of 2008. N Engl J Med 2008;358:2661-2663.

-23 McBride C, Hensley Alford S, Reid RJ, Larson EB, Baxevanis AD, Brody LC: Characteristics of users of online personalized genomic risk assessments: implications for physician-patient interactions. Genet Med 2009;11:582-587. 